\title{
Desafios enfrentados por mães no tratamento de filhos com microcefalia
}

\author{
Challenges faced by mothers in the treatment of children with microcephal \\ Desafíos que enfrentan las madres en el tratamiento de niños con microcefal
}

\begin{abstract}
Andresa Teixeira Santos ${ }^{1 *}$, Geane de Jesus Tavares ${ }^{1}$, Sheylla Nayara Sales Vieira ${ }^{1}$, Gislene de Jesus Cruz Sanches ${ }^{1}$, Franciane Neves Silva ${ }^{1}$, Geovana de Jesus Santana ${ }^{1}$, Isline Carizia Borges Sanches $^{1}$, Lays Santos França ${ }^{1}$, Mirian Souza Pereira da Silva ${ }^{1}$, Renara Meira Gomes ${ }^{1}$, Afonso Henrique Barreto da Silva ${ }^{1}$, Milane Correia Garcia ${ }^{1}$.
\end{abstract}

\section{RESUMO}

Objetivo: conhecer os desafios enfrentados por mães no tratamento de filhos com microcefalia. Métodos: trata-se de estudo descritivo-exploratório de natureza qualitativa, realizado com mães de filhos microcefálico em uma cidade de médio porte no sudoeste da Bahia. A população do estudo foi constituída de 07 mães de crianças com microcefalia de sexo e idade diferentes, cujos dados foram coletados por meio de entrevista semiestruturada, gravada com auxílio de gravador e analisada através da análise do discurso do sujeito coletivo. Resultados: os dados coletados permitiram a construção de discursos coletivos que representam o entendimento das mães sobre os desafios enfrentados no tratamento de filhos com microcefalia. A partir das ideias temáticas dos discursos analisados que responderam ao objetivo proposto, surgiram três categorias para a reflexão: "o entendimento das limitações físicas dos filhos", "dificuldade enfrentada para o atendimento com equipe multiprofissional" e a "garantia de atendimento especializado pelo SUS". Conclusão: Diante dos resultados encontrados, fica evidente que é necessário o surgimento de estratégias para que os desafios sejam enfrentados, como fortalecimento do SUS, uma rede apoio eficaz e assistência aos pacientes acometidos, para que os usuários não peregrinem dentro do sistema, também realizar uma capacitação de profissionais de saúde para lidar com a situação.

Palavras-chave: Educação em saúde, Reabilitação, Vírus zika.

\begin{abstract}
Objective: to know the challenges faced by mothers in the treatment of children with microcephaly. Methods: This is a descriptive-exploratory study of a qualitative nature, carried out with mothers of microcephalic children in a medium-sized city in the southwest of Bahia. The study population consisted of 07 mothers of children with microcephaly of different sex and age, whose data were collected through a semi-structured interview, recorded with the aid of a tape recorder and analyzed through the discourse analysis of the collective subject. Results: the collected data allowed the construction of collective discourses that represent the mothers' understanding of the challenges faced in the treatment of children with microcephaly. Based on the thematic ideas of the analyzed discourses that responded to the proposed objective, three categories emerged for reflection: "the understanding of the physical limitations of the children", "difficulty faced for attendance with multiprofessional team" and the "guarantee of specialized care by SUS". Conclusion: Given the results, it is evident that the emergence of strategies is necessary for the challenges to be faced, such as strengthening the SUS, an effective support network and assistance to affected patients, so that users do not peregrinate within the system, also perform a training of health professionals to deal with the situation.
\end{abstract}

Key words: Health education, Rehabilitation, Zika virus.

${ }^{1}$ Faculdade de Tecnologia e Ciências/FTC, Campus de Jequié. Jequié (BA), Brasil.

*E-mail: atsantos.jeq@ftc.edu.br

SUBMETIDO EM: 7/2019

ACEITO EM: 7/2019

PUBLICADO EM: $8 / 2019$

REAS/EJCH | Vol.Sup.32 | e1234 | DOI: https://doi.org/10.25248/reas.e1234.2019 Página 1 de 7 


\section{RESUMEN}

Objetivo: conocer los desafíos que enfrentan las madres en el tratamiento de niños con microcefalia. Métodos: Este es un estudio descriptivo exploratorio de naturaleza cualitativa, realizado con madres de niños microcefálicos en una ciudad de tamaño mediano en el suroeste de Bahía. La población de estudio consistió en 07 madres de niños con microcefalia de diferente sexo y edad, cuyos datos se recopilaron a través de una entrevista semiestructurada, se registraron con la ayuda de una grabadora y se analizaron mediante el análisis del discurso del sujeto colectivo. Resultados: Los datos recopilados permitieron la construcción de discursos colectivos que representan el entendimiento de las madres sobre los desafíos que enfrentan en el tratamiento de niños con microcefalia. Sobre la base de las ideas temáticas de los discursos analizados que respondieron al objetivo propuesto, surgieron tres categorías para la reflexión: "la comprensión de las limitaciones físicas de los niños", "la dificultad que enfrenta la asistencia de un equipo multiprofesional" y La "garantía de atención especializada por el SUS". Conclusión: Dados los resultados, es evidente que el surgimiento de estrategias es necesario para enfrentar los desafíos, como el fortalecimiento del SUS, una red de apoyo efectiva y asistencia a los pacientes afectados, para que los usuarios no peregrinen dentro del sistema, también realicen Una formación de profesionales de la salud para hacer frente a la situación.

Palabras clave: Educación para la salud, Rehabilitación, Virus zika.

\section{INTRODUÇÃO}

A microcefalia é uma má formação que apresenta alterações de estrutura e função do sistema neurológico, caracterizada pela medida do perímetro cefálico, realizada logo após o nascimento da criança, onde se considera um resultado menor que o esperado para o sexo e idade gestacional. Não é propriamente uma deficiência, mas pode acarretar no surgimento de uma deficiência física como consequência da agressão ao cérebro; as alterações podem ocorrer porque o cérebro precisa de espaço para se desenvolver, mas o crânio restringe (OLIVEIRA MC e SÁ SMA, 2017).

A condição da microcefalia repercute negativamente no desenvolvimento da criança, a evolução neuropsicomotora desta costuma ser lento, o comprometimento intelectual na maioria dos casos é severo e - comportamento dessas crianças costuma ser atípico, podendo evidenciar a presença de choro e irritabilidade contínua, entre outros acometimentos, $O$ que leva os pais a ser principal agente do cuidado à criança com deficiência ou com necessidades especiais (BERTUOLI N e OLIVEIRA AKC, 2016).

No que se refere à sua etiologia, a microcefalia é subdividida em dois grupos principais: a microcefalia primária e a microcefalia secundária. A microcefalia primária está presente no nascimento, com a identificação do baixo perímetro cefálico do bebê, como consequência de uma diminuição na espessura do córtex cerebral, malformações ou outras alterações neurológicas significativas Já à microcefalia secundária, está relacionada a causas secundárias, variando a gravidade da malformação, as funções cerebrais são pouco desenvolvidas devido a um grande número de agentes nocivos que atingem o feto no útero ou o lactente durante os períodos de rápido crescimento cerebral (BERTUOLI N e OLIVEIRA AKC, 2016).

No Brasil, no período de 2010 a 2014, uma média de 156 casos de microcefalia era registrada anualmente, no Sistema de Informações sobre Nascidos Vivos (Sinasc). Porém, ao final do ano de 2015, surgiram os primeiros relatos de várias crianças nascidas com microcefalia em Unidades de Saúde do Estado do Pernambuco, na região Nordeste do Brasil, onde em 2016 após estudos investigativos foi concluído que o vírus zika seria o principal responsável dessa epidemia que não se limitava mais só na região nordeste, mas em todo Brasil (REIS JC, et al, 2018).

Até 27 de fevereiro de 2016, foram notificados 5.909 casos de microcefalia, sendo 641 confirmados para microcefalia ou alteração do SNC sugestivos de infecção congênita, dos 5.909 casos, 139 evoluíram para óbito fetal ou neonatal e 96 permanecem em investigação, 31 foram confirmados para microcefalia e 12 foram descartados (SOUSA PSA, et al, 2016). Frente ao número elevado de casos notificados abruptamente, foi observado em todo país, mães e famílias completamente impactadas sobre o que fazer como cuidar e onde procurar auxílio médico para suas crianças nascidas com microcefalia, pois o sistema de saúde não estava preparado para atender toda essa demanda (VEIGA SA, et al, 2017). 
A população brasileira, assim como o sistema de assistência à saúde de uma maneira geral, encontra-se diante de um grande desafio, que e o de entender o potencial do novo teratógeno e desvendar os mecanismos patogênicos do ZIK-V (vírus zika), para o enfrentamento preventivo e reconhecimento amplo e espectro de manifestações clínicas, principalmente o impacto no desenvolvimento cognitivo-comportamental, para assim poder elaborar programas de intervenção visando o melhor cuidado das crianças e das famílias envolvidas (BRUNONI D, et al., 2016).

Frente ao exposto e considerando-se os altos índices de incidência de infecções pelo vírus zika no Brasil e a sua relação com casos de microcefalia em recém-nascidos, objetivou-se conhecer os desafios enfrentados por mães no tratamento de filhos com microcefalia.

\section{MÉTODOS}

O estudo possui abordagem qualitativa e caráter descritivo-exploratório. Aconteceu nas residências de mães de crianças com microcefalia cadastradas no centro de referência localizada na zona urbana de um município do sudoeste da Bahia, no período de julho a agosto de 2017, após a análise de prontuários médicos de crianças acompanhadas no centro de referência da Associação de Pais e Amigos de Excepcionais (APAE). As pesquisadoras convidaram as mães a participarem do estudo na própria unidade e nas residências, ficando acordado entre as pesquisadoras e mães, entre as residências e unidade o melhor local para acontecer o estudo.

A amostra foi composta de mães de crianças com diagnóstico clínico de microcefalia associada à infecção materna por vírus Zika. As visitas foram previamente agendadas pelas pesquisadoras. Ressalta-se que foram excluídos os participantes que não compareceram nas unidades para o agendamento da visita na residência. Assim, o estudo teve como amostra 7 mães que atenderam aos critérios de inclusão e que aceitaram participar da pesquisa, assinando os Termos de Consentimento Livre e Esclarecido (TCLE) com autorização de gravação e uso de áudio.

Utilizou-se como método de investigação e coleta de dados às entrevistas e um roteiro semiestruturados. As entrevistas foram norteadas pelo roteiro composto por duas partes: a primeira, com questões objetivas referentes ao perfil sócio demográfico das participantes; e a segunda, com questões abertas. Todos os dados relevantes dos discursos eram armazenados através de recursos de áudio em gravação e em diários de campo para análise posterior.

Para a análise e interpretação dos dados, foi utilizada a técnica do Discurso do Sujeito Coletivo-DSC. O diferencial da metodologia do DSC é que a cada categoria estão associados os conteúdos das opiniões de sentido semelhante presentes em diferentes depoimentos, de modo a formar com tais conteúdos um depoimento síntese, redigido na primeira pessoa do singular, como se tratasse de uma coletividade falando na pessoa de um indivíduo (LEFEVRE F e LEFEVRE AMC, 2014). O estudo seguiu as determinações éticas da Resolução no 466/12 do Conselho Nacional de Saúde sobre pesquisa envolvendo seres humanos.

\section{RESULTADOS E DISCUSSÃO}

As mães entrevistadas se caracterizam por terem idade entre 24 e 35 anos. No que diz respeito ao estado civil, $57,14 \%$ convivem com os companheiros, sendo solteiras ou divorciadas $42,86 \%$. O grau de instrução variou de ensino fundamental completo ao Ensino Médio completo. Apenas $28,57 \%$ das mães trabalham. A renda média mensal variou entre 1 a 3 salários mínimos e 71,42 \% possuem o Benefício de Prestação Continuada (BPC), que é tida como uma renda de um salário-mínimo que os idosos e deficientes recebem quando os mesmos não têm condições de se manterem sozinho ou não podem ser mantidos pela família. Em relação aos números de filhos por mães variou de três a quatros filhos. Os depoimentos evidenciaram mães não tabagistas, consume bebida alcoólica socialmente e não pratica atividade física.

Os dados coletados permitiram a construção de discursos coletivos que representam o entendimento das mães sobre os desafios enfrentados no tratamento de filhos com microcefalia. A seguir foram apresentadas as ideias centrais retiradas das falas das entrevistadas e os discursos construídos a partir destas. 
As respostas permitiram a construção de seis discursos, nos quais se busca entender os desafios encontrados diariamente pelas mães no tratamento de seus filhos.

\section{Limitações físicas: saberes e desconhecimentos de mães}

Quando questionadas sobre o conhecimento que as mães têm sobre as patologias dos seus filhos as falas apresentaram discursos dicotômicos.

\section{Discurso 1 - Conhecimento e desconhecimento das limitações físicas e cognitivas dos filhos}

[..]sim, vai passando o tempo e vou conhecendo essas limitações físicas dele, claro que a gente ver que se meu filho for se relacionar com uma criança de 2 anos que não tem a micro, é bem diferente, tudo nele é mais lento[...]

O coletivo das mães demonstra que as mesmas possuem conhecimento sobre as limitações que seus filhos apresentam como consequência a microcefalia, e expressam que reconhecem as barreiras existentes no que tange o relacionamento de seus filhos com outras crianças da mesma faixa etária, que não possuem a comorbidade.

Os familiares entram em contato com a temática da deficiência de diferentes maneiras. Durante o prénatal, por exemplo, o médico pode encontrar indícios clínicos de que algo não está bem. Por um lado, são comuns, nessa fase, indicativos médicos que levantam a suspeita de malformações, infecções ou síndromes que levam à deficiência ou em consultas subsequentes com outros profissionais (LEVANDOWSKI M e CARRILHO L, 2014).

Entretanto ao longo da pesquisa uma pequena parcela das mães desconhecia as limitações que a patologia dos seus filhos apresenta, relacionado ao seu crescimento e desenvolvimento, e referirem que não entendem sobre a comorbidades, como está sendo demonstrado na fala abaixo.

\section{[...] não entendo, não sei quase nada [...].}

Pois compreendemos que uma grande parte dos profissionais da saúde, principalmente os médicos, em geral, não são capacitados para lidar com a deficiência, principalmente quando se defrontam com um bebê com suspeita diagnóstica de alguma patologia ou síndrome. Os médicos raramente esclarecem ou informam aos familiares sobre as possibilidades de desenvolvimento da criança, a possibilidade de superação das dificuldades ou sobre os locais onde os recursos são oferecidos (SANCHES LAS e FIAMENGHI-JR GA, 2011).

As mães precisam de ajuda, orientação, informação e compreensão, para que possam ajustar-se e realizar satisfatoriamente a sua função materna. É imprescindível que a família estabeleça conhecimentos sobre as necessidades especiais de seus filhos, construa padrões de enfrentamento dos sentimentos e das necessidades como um todo, na tomada de decisões e na busca dos recursos para o bem-estar da criança e da família (SANTOS LS, et al., 2017).

Os casos de microcefalia em sua maioria têm associado aos seus sinais e sintoma as alterações motoras e cognitivas que alteram de acordo com o grau de acometimento cerebral. O comprometimento varia para cada paciente pois depende muito da área e da extensão do cérebro que foi lesionado pela doença, podendo a criança apresentar diversas dificuldades como um declínio no desenvolvimento neuropsicomotor (DNPM), pode apresentar também déficits auditivos, físicos, intelectuais, cognitivos e visuais o que leva a compreensão das dificuldades enfrentadas pela família no tratamento e reabilitação dos seus filhos (NORBERT AAF, et al., 2016). As alterações mais comumente associadas à microcefalia estão relacionadas ao déficit intelectual e outras condições, que incluem: irritabilidade; convulsões; epilepsia; paralisia cerebral; atraso no desenvolvimento de linguagem e/ou motor; desordens oftalmológicas, cardíacas, renais e do trato urinário; entre outras (SÁ FE, et al., 2017).

No geral, as crianças que tem microcefalia apresentam atraso no desenvolvimento neuropsicomotor com acometimento motor e cognitivo relevante e, em alguns casos as funções sensitivas (audição e visão) também são comprometidas e o sistema respiratório também é acometido devido às alterações do tônus muscular, 
gerando uma pobreza dos movimentos e um enfraquecimento dos músculos ventilatórios, promovendo um déficit nas trocas gasosas (SOUSA ETC, et al., 2017).

\section{Os impasses vivenciados durante $o$ atendimento com a equipe multiprofissional}

No discurso, que versa sobre o serviço de atendimento multiprofissional e intervenção precoce com crescimento e desenvolvimento das crianças, os discursos parentais referenciam o desejo de melhores condições de saúde transporte e atendimento de profissionais com especialidades como demonstra os discursos a seguir.

\section{Discurso 3- Atendimento com a equipe multiprofissional: Facilidades e dificuldades}

[...] acho que não tem nenhuma dificuldade lá na APAE, eu não falo nada de lá, atendem muito bem eu nunca tive problemas lá, todos os exames que preciso marcam inclusive médico. [...]

O coletivo das mães acima relata que não possuem dificuldades no processo de atendimento de seus filhos, e descreve as facilidades presentes durante 0 atendimento nas instituições a qual são direcionadas.

O discurso demonstra que às facilidades encontradas pelo coletivo, em relação ao atendimento das crianças acometidas pela microcefalia está intimamente relacionada a uma assistência prestada por instituições não governamentais.

A participação expressiva de instituições não governamental objetiva a complementação e aprimoramento da assistência prestada à população, bem como a condição e a natureza do cuidado prestado a crianças com necessidades especiais, e mesmo com as dificuldades, a formação de uma rede de apoio voltada para a inclusão e a acessibilidade da criança, auxilia na aquisição de sua independência, qualificando o seu viver (PINTANEL AC, et al, 2013).

A Constituição Federal estabelece que a "família é à base da sociedade" (Art. 226) e que, portanto, compete a ela, juntamente com o Estado, a sociedade em geral, "assegurar à criança e ao adolescente o exercício de seus direitos fundamentais" tais como promoção e proteção dos direitos humanos e apoio às crianças e aos adolescentes com deficiência (BRASIL, 2006).

Sabe-se que o acompanhamento adequado contribui para a geração de indivíduos com maior senso crítico, aptos a enfrentarem dificuldades, tornarem-se adolescentes, jovens e adultos sadios e socialmente produtivos (SIMIÃO CKS, et al., 2017).

Entretanto a coletividade também expressa a as diversas dificuldades que as mães enfrentam na busca pelo atendimento com a equipe multiprofissional que os filhos necessitam, e relatam tais dificuldades, sobre o transporte oferecido, o atendimento pela fisioterapia que é deficiente e a falta de médicos nas diversas especialidades.

[...] Sim, há uma falta de transporte, deficiência da prefeitura e do estado em oferecer uma, eco terapia, sendo que se for é particular, tinha que ter uma parceria com a prefeitura, uma hidroterapia, que não tem, e o que oferecido na fisioterapia é muito pouco, se não tiver outros recursos nossos filhos sempre vão ficar para traz sem algumas especialidades médicas, como: pediatria, fonoaudióloga que não encontro, entre todas as dificuldades, a mais difícil é encontrar um neurologista pediatra. [...]

O discurso demonstra um entendimento sobre a necessidade de atendimento diferenciado e especializado para os filhos, no entanto esbarram em socioeconômicas, onde a grande maioria das famílias encontra dificuldades no custo dos transportes e mantimentos das crianças. $O$ atendimento especializado para estimulação precoce possui sessões reduzidas que não ocorrem conforme a orientação dos protocolos e manuais vigentes, acorrendo atendimentos uma vez por semana, enquanto o recomendado é três vezes semanal, e a falta de profissionais capacitados dispostos a trabalhar com patologias específicas relacionadas ao Neurodesenvolvimento. 
A família encontra, de maneira geral, dificuldades para encontrar apoio social. Para ela, a medicina salvou a vida de sua criança, mas a sociedade não está preparada para acolhê-la. No que diz respeito à saúde, não é fácil obter os recursos de que a criança precisa e nem sempre a cidade os oferece, tendo a família de buscar tratamento e acompanhamento fora do município (ANDRADE MB, et al, 2011).

\section{O SUS e suas demandas diante da microcefalia}

Sabe-se que, as crianças acometidas por microcefalia possuem necessidades especiais, assim necessitam de cuidados direcionados e qualificados. Muitas das famílias que possuem filhos acometidos pela patologia em questão são usuários do Sistema Único de Saúde - SUS. Diante disso faz necessário políticas direcionadas e cuidados especializados ofertados por esse sistema de saúde.

Nesse sentindo questionou-se sobre o atendimento prestado pelo SUS a crianças diagnosticadas com microcefalia. Do questionamento emergiu dois discursos, que seguem.

\section{Discurso 5 - Satisfação e insatisfação no atendimento especializado}

[...] sim, desde a época que ela nasceu, eu uso o SUS, no posto eles a atendem mensalmente, em outros estabelecimentos, dia de segunda, duas vezes por semana, todo mês, o serviço é de qualidade. [...]

O discurso acima descreve a satisfação do coletivo relacionada ao atendimento ofertado pelo SUS aos seus filhos. E especifica os dias de atendimento estabelecidos. Sobre o atendimento prestado pelo sistema único de saúde - SUS, apesar da satisfação demonstrada pelas mães, é compreensivo que as mesmas não reconheçam ou encontrem dificuldade na reabilitação dos seus filhos, pois é visto que os pais precisam receber informações a respeito do estado de saúde do filho, diagnóstico, tratamento e prognóstico, os quais afirmam que as informações fornecidas pelos profissionais parecem estar sendo suficientes, mas algumas mães não dispõem de conhecimentos sobre a patologia e/ou deficiência tornando a compreensão, mas difícil no que se refere $o$ atendimento prestado.

A equipe multidisciplinar, fisioterapeutas, terapeutas ocupacionais, assistentes sociais, médicos, psicólogos e enfermeiros exercem um papel fundamental, pois por meio das informações que eles transmitem para a família elas passam a buscar informações, aprender por meio de outras fontes, devido à necessidade de conviver com a criança. Pois existem protocolos de atendimento específicos, todas as crianças com microcefalia deverão manter consultas de puericultura nas Unidades Básicas de Saúde (UBS). Também deverão ser acompanhados em serviços especializados para estimulação essencial, já que a criança tem a lesão e há o quadro neurológico. É necessário estimular as percepções sensoriais, os movimentos e o posicionamento da criança, a coordenação motora, o brincar, a socialização e a cognição (BRASIL, 2016).

Quanto mais precoce e objetiva for essa intervenção, maior e melhor serão as chances da criança se desenvolver em suas potencialidades e com menor déficit residual. Contudo, a estimulação é necessária para melhorar a condição de vida o que leva a necessidade da busca por melhores tratamentos, que possa contribuir com desenvolvimento (WAJNSZTEJN R, 2016).

Durante a entrevista o coletivo cita as insatisfações que existe com o atendimento prestado, e especifica as falhas existentes. Em decorrência da recente epidemia dos casos de microcefalia no cenário brasileiro, entende-se como necessário e urgente conhecer as necessidades presentes no dia- dia das crianças, assim como a importância dos profissionais na compreensão e atuação na dinâmica familiar, com olhar para as diferentes necessidades maternas vivenciadas em sua rotina diária junto à criança, para assim poder oferecer um tratamento adequado a cada criança de acordo com suas necessidades, mostrando as possibilidades positivas, e não somente os aspectos negativos.

[...] uma coisa que o prefeito e o secretário de saúde, deveriam olhar com um olhar diferenciado, para esses casos de microcefalia, que são casos novos, os profissionais deveriam tomar cursos, para atender essas crianças, justamente porque, não sei, acho que falta muito ainda pra fazer pelos nossos filhos. [...] 
Para que assim possa promover autoconfiança e proporcionar apoio adequado para um melhor desempenho do dia a dia familiar podendo intensificar o empoderamento dos pais, propiciando bem-estar, o enfrentamento saudável da condição de vida, melhora no acesso e no apoio aos serviços necessários e facilidades na prestação de melhores serviços para as famílias (SOUZA SEP, 2017).

Pois todas as crianças acometidas com microcefalia ao nascer podemos considerar que é especial e única, e sua a avaliação e tratamento deve ser holística e criteriosa tendo como base o que deve ser estimulado para que assim seja possível diminuir a probabilidade de ocorrer atrasos no seu desenvolvimento neuropsicomotor (NORBERT AAF et al., 2016).

\section{CONSIDERAÇÕES FINAIS}

Diante dos resultados encontrados, verificou-se que os desafios encontrados pelas mães de crianças com microcefalia para o tratamento de seus filhos, estão na dificuldade de transporte para encaminhamento nos dias de consultas e terapias, profissionais com especialidades e conhecimento técnico cientifico sobre a patologia, dificuldade de agendamento para atendimento especializado conforme o apregoado pelos protocolos do Ministério da Saúde. Sendo assim, para que esses desafios sejam enfrentados, cabem algumas propostas, entre elas fortalecimento do Sistema Único de Saúde - SUS, reforço de uma rede apoio e assistência aos pacientes acometidos, para que os usuários não peregrinem dentro do sistema, investir de forma arrojada no controle de vetores, e capacitação de profissionais de saúde e participação da família.

\section{REFERÊNCIAS}

1. ANDRADE MB, et al. Paralisia cerebral: estudo sobre o enfrentamento familiar. Reme, Rev. Min. Enferm.; 15(1):86-96, 2011.

2. BERTUOLI N, OLIVEIRA AKC. Microcefalia: experiências e expectativas junto à realidade materna. Dissertação (Graduação em Terapia Ocupacional) - Centro de Ciências da Saúde. Universidade Federal da Paraíba (UFPB). João Pessoa, 2016.

3. BRASIL. Conselho Nacional dos Direitos da Criança e do Adolescente. Conselho Nacional de Assistência Social Plano Nacional de Promoção, Defesa e Garantia do Direito de Crianças e Adolescentes à Convivência Familiar e Comunitária. 2006.

4. Brasil. Ministério da Saúde. Secretaria de Atenção à Saúde. Protocolo de atenção à saúde e resposta à ocorrência de microcefalia relacionada à infecção pelo vírus zika. Brasília: Ministério da Saúde, 2016.

5. BRUNONI D, et al. Microcefalia e outras manifestações relacionadas ao vírus Zika: impacto nas crianças, nas famílias e nas equipes de saúde. Ciênc. Saúde coletiva; 21(10):3297-3302, 2016.

6. LEFEVRE F, LEFEVRE AMC. Discurso do sujeito coletivo: representações sociais e intervenções comunicativas. Texto contexto - enferm; 23(2): 502-507, 2014.

7. LEVANDOWSKI M, CARRILHO L. Expectativa dos pais de crianças com patologias neurológicas em relação à fisioterapia. Saúde Integrada, 2014.

8. NORBERT AAF, et al. A importância da estimulação precoce na microcefalia. Salão do Conhecimento [S.I.], 2016.

9. OLIVEIRA MC, SÁ SM. A Experiência Parental após o Diagnóstico da Microcefalia por Zika Vírus: Um Estudo de Caso. Revista Pesquisa em Fisioterapia; 7(4):64-70, 2017.

10. PINTANEL AC, et al. Facilidades e dificuldades da criança com deficiência visual para o exercício da independência: percepções da família. Rev. enferm. UFPE; 7(1):119-27, 2013.

11. REIS J.C, et al. Abordagem da terapia ocupacional a bebês com microcefalia: uma experiência no estágio curricular. Rev. Interinst. Bras. Ter. Ocup; 2(1):212-227, 2018.

12. SÁ FE, et al. Produção de sentidos parentais no cuidado de crianças com microcefalia por vírus zika. Rev Bras Promoç Saúde; 30(4):1-10, 2017.

13. SANCHES LAS, FIAMENGHI-JR GA. Relatos maternos sobre o impacto do diagnóstico da deficiência dos filhos. Cad. Saúde colet.;19(3), 2011.

14. SANTOS LS, et al. A participação da família no trabalho de reabilitação da criança com microcefalia. Ciências Biológicas e de Saúde Unit; 4(2):189-202, 2017.

15. SIMIÃO CKS, et al. Atenção integrada às doenças prevalentes na infância: prática do enfermeiro. Revista de Enfermagem UFPE, [S.I.]; 11(12):5382-5390, 2017.

16. SOUSA ETC, et al. A microcefalia por possível infecção intrauterina do zika vírus e suas complicações no sistema respiratório: um relato de caso. CONBRACIS. 2017.

17. SOUSA PSA, et al. Microcephaly zika and viruses: a systematic review. Rev Enferm UFPI; 5(4):51-4, 2016.

18. SOUZA SEP. Reabilitação para crianças com síndrome congênita do zika vírus: percepção de profissionais de saúde em uma unidade de Vitoria de Santo Antão, Pernambuco. TCC (Graduação em Saúde Coletiva) - Universidade Federal de Pernambuco, Vitória de Santo Antão, 2017.

19. VEIGA SA, et al. Assistência de enfermagem à criança com microcefalia. Múltiplos Acessos; 2(2), 2017.

20. WAJNSZTEJN, R. Síndrome congênita do zika. II Painel Latino-Americano Arboviroses de Importância para Saúde Humana. 2016. 